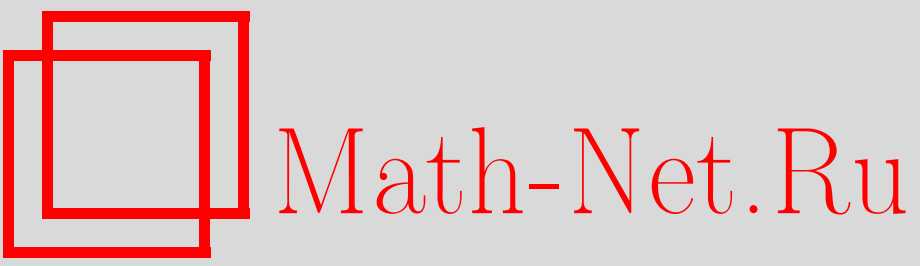

Л. Ф. Блажиевский, Об одном представлении статистической суммы с помощью грассмановых переменных, ТМФ, 2001, том 126, номер 2, 301-310

DOI: https://doi.org/10.4213/tmf432

Использование Общероссийского математического портала Math-Net.Ru подразумевает, что вы прочитали и согласны с пользовательским соглашением

http://www.mathnet.ru/rus/agreement

Параметры загрузки:

IP : 107.22 .136 .117

26 апреля 2023 г., 17:57:41 
ТЕОРЕТИЧЕСКАЯ

И МАТЕМАТИЧЕСКАЯ

ФИЗИКА

Том 126, № 2

февраль, 2001

(C) 2001 г.

Л. Ф. Блажиевский*

\section{ОБ ОДНОМ ПРЕДСТАВЛЕНИИ СТАТИСТИЧЕСКОЙ СУММЫ С ПОМОШЬЮ ГРАССМАНОВЫХ ПЕРЕМЕННЫХ}

Предложена формула для классической статистической суммы $Z_{N}$, не требующая знания гамильтоновой функции системы. Показано, что в общем случае можно избежать процедуры перехода к каноническим переменным $(\mathbf{p}, \mathbf{x})$, если расширить пространство лагранжевых переменных $(\mathbf{v}, \mathbf{x})$ введением "дополнительных скоростей" $\overline{\mathbf{u}}, \mathbf{u}$, являющихся образующими грассмановой алгебры. В этом пространстве статсумма $Z_{N}$ представляется интегралом от некоторого распределения гиббсовского типа, явный вид которого определяется лагранжианом системы. Рассчитана статистическая сумма модельной системы, описываемой лагранжианом Дарвина.

Как известно, традиционная формулировка статистической механики, основываюшаяся на распределении Гиббса и уравнении Лиувилля, предполагает использование для описания системы гамильтоновых переменных. Однако применение этой схемы при исследовании слаборелятивистских (иначе постньютоновских) систем сушественно усложняется нетривиальностью перехода к этим переменным. В постньютоновском приближении обычно известны функции Лагранжа $L(\mathbf{v}, \mathbf{x})$. Для них характерна сложная зависимость от скоростей частиц $\mathbf{v}$ (укажем в этой связи на лагранжианы Дарвина, Эйнштейна-Инфельда-Гоффмана [1], Кеннеди [2], Гайды [3]). Переход к гамильтоновым переменным $(\mathbf{p}, \mathbf{x})$ связан с разрешением системы уравнений $\mathbf{p}=\partial L(\mathbf{v}, \mathbf{x}) / \partial \mathbf{v}$ относительно скоростей v. Это можно сделать только приближенно, учитывая постньютоновские члены по теории возмущений. Использовав преобразование Лежандра, несложно показать, что если лагранжиан системы имеет вид $L(\mathbf{v}, \mathbf{x})=L_{0}(\mathbf{v}, \mathbf{x})+U(\mathbf{v}, \mathbf{x})$ и $U \ll L_{0}$, то в первом приближении гамильтониан определяется формулой

$$
H(\mathbf{p}, \mathbf{x})=H_{0}(\mathbf{p}, \mathbf{x})-U\left(\frac{\partial H_{0}}{\partial \mathbf{p}}, \mathbf{x}\right)
$$

где $L_{0}(\mathbf{v}, \mathbf{x})$ и $H_{0}(\mathbf{p}, \mathbf{x})$ - соответственно функции Лагранжа и Гамильтона невозмущенной системы.

Постньютоновские гамильтонианы вида (1) можно использовать в задачах как классической, так и квантовой механики. В статистической механике ситуация оказывается

\footnotetext{
* Львовский национальный университет, Львов, Украина
} 
более сложной. Исходные соотношения статистической механики имеют физический смысл (т.е. результаты, полученные на их основе, согласуются с феноменологической термодинамикой) только в термодинамическом пределе, когда число частиц $N$ и объем системы $V$ связаны соотношением $N / V=$ const при $N \rightarrow \infty, V \rightarrow \infty$. Это условие необходимо учитывать при приближенном разрешении системы уравнений $\mathbf{p}=\partial L / \partial \mathbf{v}[4]$. В частности, если взаимодействие дальнодействующее, то можно показать, что расчет термодинамических функций на основе формулы (1) приводит к расходящимся выражениям. Это видно уже из первых работ [5], [6] по статистической термодинамике слаборелятивистской плазмы, в которых использовался гамильтониан Дарвина [1]. Для устранения расходимостей авторам пришлось прибегнуть к искусственному “обрезанию" соответствующих интегралов. Однако расходимости компенсируются, если в выражении для гамильтониана учесть члены высшего порядка, отброшенные в формуле (1). Впервые на это обстоятельство было обрашено внимание Трубниковым и Косачевым в статье [7]. Впоследствии эти вопросы неоднократно обсуждались в литературе. Например, в работах [8]-[16] можно найти аргументы как за, так и против приближения (1).

В этой связи представляется уместным развитие формализма, в котором исходные соотношения статистической механики записывались бы непосредственно в лагранжевых переменных. В частности, автором показано [17], что статистическую матрицу плотности можно представить фейнмановским интегралом по траекториям, содержащим действие в лагранжевой форме, если только этот интеграл должным образом регуляризовать. В данной работе предлагается другой подход, используюший грассмановы переменные.

Ограничимся рассмотрением классических равновесных систем. Статистическую сумму

$$
Z_{N}=\int d \Gamma_{N} e^{-\beta H(\mathbf{p}, \mathbf{x})}, \quad d \Gamma_{N}=\prod_{j} \frac{d \mathbf{p}_{j} d \mathbf{x}_{j}}{(2 \pi \hbar)^{3}}
$$

заменой переменных $p_{j}^{\mu}=\partial L / \partial v_{j}^{\mu}$ можно преобразовать к виду

$$
Z_{N}=\int d \widetilde{\Gamma}_{N} e^{-\beta E(\mathbf{v}, \mathbf{x})}
$$

где

$$
E(\mathbf{v}, \mathbf{x})=\sum_{j} v_{j}^{\mu} \frac{\partial L}{\partial v_{j}^{\mu}}-L, \quad d \widetilde{\Gamma}_{N}=\operatorname{det}\left|\frac{\partial^{2} L}{\partial v_{j}^{\mu} \partial v_{l}^{\nu}}\right| \prod_{j} \frac{d \mathbf{v}_{j} d \mathbf{x}_{j}}{(2 \pi \hbar)^{3}}
$$

$\beta^{-1}$ - температура; греческие индексы принимают значения $1,2,3$, их повторение подразумевает свертку. Хотя формулы $(3),(4)$ и выражают статистическую сумму через лагранжиан системы, однако при вычислении определителя возникают те же осложнения, что и при определении гамильтоновой функции. Нам желательно иметь для $Z_{N}$ выражение, расчет которого можно было бы осуществить такими же методами, какие обычно применяются для приближенного вычисления классической статистической суммы нерелятивистских систем. 
Воспользуемся известным [18] интегральным преобразованием на грассмановой алгебре

$$
\begin{aligned}
& \int\left(\prod_{c} d \bar{z}_{c} d z_{c}\right) \exp \left\{-\sum_{a, b} \bar{z}_{a} z_{b} A_{a b}+\sum_{a}\left(\bar{\eta}_{a} z_{a}+\bar{z}_{a} \eta_{a}\right)\right\}= \\
& =\operatorname{det}\left|A_{a b}\right| \exp \left\{\sum_{a, b} \bar{\eta}_{a} \eta_{b} A_{a b}^{-1}\right\},
\end{aligned}
$$

здесь $A_{a b}$ и $A_{a b}^{-1}$ - элементы симметричной матришы $A$ и обратной к ней. Все образуюшие $\bar{z}_{a}, z_{a}, \bar{\eta}_{a}, \eta_{a}$ подчинены антикоммутационным перестановочным соотношениям, например $z_{a} z_{b}+z_{b} z_{a}=0, \bar{z}_{a} \eta_{a}+\eta_{a} \bar{z}_{a}=0$. Символы $d \bar{z}_{a}, d z_{b}$ также антикоммутируют друг с другом и с образуюшими. Таким же условиям подчинены и символы производных, т.е. дифференцирование осушествляется по правилу

$$
\frac{\partial}{\partial \eta_{a}} \eta_{b}=\delta_{a b}-\eta_{b} \frac{\partial}{\partial \eta_{a}}
$$

где $\delta_{a b}$ - символ Кронекера. Однократные интегралы определены равенствами

$$
\int d z_{a}=\int d \bar{z}_{a}=0, \quad \int d z_{a} z_{a}=\int d \bar{z}_{b} \bar{z}_{b}=1 .
$$

Ниже нам понадобятся интегралы вида

$$
\int \prod_{c} d \bar{z}_{c} d z_{c} \exp \left\{-\sum_{a, b} \bar{z}_{a} z_{b} A_{a b}\right\} I\left(\ldots \bar{z}_{d}, \ldots z_{s}\right) \equiv I\left[\ldots \bar{z}_{d}, \ldots z_{s}\right] .
$$

Для их вычисления можно воспользоваться дифференцированием выражения (5) по образуюшим $\bar{\eta}_{a}, \eta_{a}$. Получим

$$
I\left[\ldots \bar{z}_{d}, \ldots z_{s}\right]=\left.\operatorname{det}\left|A_{a b}\right| I\left(\ldots,-\frac{\partial}{\partial \eta_{d}}, \ldots, \frac{\partial}{\partial \bar{\eta}_{s}}, \ldots\right) \exp \left\{\sum_{a, b} \bar{\eta}_{a} \eta_{b} A_{a b}^{-1}\right\}\right|_{\bar{\eta}=\eta=0} .
$$

В частности,

$$
\begin{gathered}
I\left[\bar{z}_{d}\right]=I\left[z_{s}\right]=0, \quad I\left[z_{s} z_{d}\right]=I\left[\bar{z}_{s} \bar{z}_{d}\right]=0 \\
I\left[\bar{z}_{d} z_{s}\right]=-\operatorname{det}|A| A_{d s}^{-1}=-I\left[z_{s} \bar{z}_{d}\right] .
\end{gathered}
$$

В нашем случае следует ввести систему векторных образуюших $\overline{\mathbf{u}}_{1}, \ldots, \overline{\mathbf{u}}_{N}, \mathbf{u}_{1}, \ldots$ $\ldots, \mathbf{u}_{N}$, каждая из которых имеет три компоненты $\bar{u}_{j}^{\mu}, u_{j}^{\mu}$. Тогда, как это видно из (5), можно записать

$$
\begin{gathered}
\operatorname{det}\left|\frac{\partial^{2} L}{\partial v_{j}^{\mu} \partial v_{l}^{\nu}}\right|=\int\left(\prod_{i} \frac{1}{\beta^{3}} d \overline{\mathbf{u}}_{i} d \mathbf{u}_{i}\right) \exp \left\{-\beta \sum_{j, l} \bar{u}_{j}^{\mu} u_{l}^{\nu} \frac{\partial^{2} L}{\partial v_{j}^{\mu} \partial v_{l}^{\nu}}\right\}, \\
d \overline{\mathbf{u}}_{j} d \mathbf{u}_{j}=d \bar{u}_{j}^{1} d u_{j}^{1} d \bar{u}_{j}^{2} d u_{j}^{2} d \bar{u}_{j}^{3} d u_{j}^{3} .
\end{gathered}
$$


Учтем также, что лагранжианы систем с парным взаимодействием частиц имеют вид

$$
L=\sum_{j} L_{j}^{0}+\frac{1}{2} \sum_{j \neq l} L_{j l}
$$

Использовав соотношения (7), (8) в формулах (3), (4), найдем, что статистическая сумма $Z_{N}$ задается формулами

$$
Z_{N}=\int d \gamma_{N} e^{-\beta \widetilde{E}(\overline{\mathbf{u}}, \mathbf{u}, \mathbf{v}, \mathbf{x})}, \quad d \gamma_{N}=\prod_{j} d \gamma(j)=\prod_{j} \frac{d \overline{\mathbf{u}}_{j} d \mathbf{u}_{j} d \mathbf{v}_{j} d \mathbf{x}_{j}}{(2 \pi \hbar \beta)^{3}},
$$

где

$$
\begin{aligned}
\widetilde{E}= & \sum_{j} \widetilde{E}_{j}^{0}+\frac{1}{2} \sum_{j \neq l} \widetilde{E}_{j l}, \\
\widetilde{E}_{j}^{0}= & v_{j}^{\mu} \frac{\partial L_{j}^{0}}{\partial v_{j}^{\mu}}-L_{j}^{0}+\frac{\partial^{2} L_{j}^{0}}{\partial v_{j}^{\mu} \partial v_{j}^{\nu}} \bar{u}_{j}^{\mu} u_{j}^{\nu}, \\
\widetilde{E}_{j l}= & v_{j}^{\mu} \frac{\partial L_{j l}}{\partial v_{j}^{\mu}}+v_{l}^{\mu} \frac{\partial L_{j l}}{\partial v_{l}^{\mu}}-L_{j l}+\frac{\partial^{2} L_{j l}}{\partial v_{j}^{\mu} \partial v_{l}^{\nu}}\left(\bar{u}_{j}^{\mu} u_{l}^{\nu}+\bar{u}_{l}^{\nu} u_{j}^{\mu}\right)+ \\
& +\frac{\partial^{2} L_{j l}}{\partial v_{j}^{\mu} \partial v_{j}^{\nu}} \bar{u}_{j}^{\mu} u_{j}^{\nu}+\frac{\partial^{2} L_{j l}}{\partial v_{l}^{\mu} \partial v_{l}^{\nu}} \bar{u}_{l}^{\mu} u_{l}^{\nu} .
\end{aligned}
$$

Полученные соотношения выражают статистическую сумму через лагранжиан системы. Мы избежали процедуры перехода к каноническим переменным $(\mathbf{p}, \mathbf{x})$ (как и вычисления определителя в (4)), расширив фазовое пространство лагранжевых переменных $(\mathbf{v}, \mathbf{x})$ введением "дополнительных скоростей" частиц $\overline{\mathbf{u}}, \mathbf{u}$. Подобно классической канонической теории в этом пространстве статистическая сумма представляется интегралом от ненормированного "распределения Гиббса" $e^{-\beta \widetilde{E}(\gamma)}, \gamma=\{\overline{\mathbf{u}}, \mathbf{u}, \mathbf{v}, \mathbf{x}\}$. При этом, как и в канонической теории, мера интегрирования в пространстве переменных $\gamma$ является произведением одночастичных мер. Сушественно также, что выражения (10), определяюшие "энергию" $\widetilde{E}$, квадратичны по грассмановым переменным. Легко проверить, что такие квадратичные формы коммутируют между собой, вследствие чего выполняются равенства

$$
e^{-\beta \widetilde{E}}=\exp \left(-\beta \sum_{j} \widetilde{E}_{j}^{0}\right) \exp \left(-\frac{\beta}{2} \sum_{j \neq l} \widetilde{E}_{j l}\right)=\left(\prod_{j} e^{-\beta \widetilde{E}_{j}^{0}}\right)\left(\prod_{j<l} e^{-\beta \widetilde{E}_{j l}^{0}}\right) .
$$

Поэтому статсумму $Z_{N}$ можно представить в виде

$$
Z_{N}=Z_{0}^{N} \int\left(\prod_{j} d \gamma(j) \varphi_{j}\right) \prod_{j<l}\left(1+F_{j l}\right) \equiv Z_{0}^{N} Q_{N}
$$

где

$$
F_{j l}=e^{-\beta \widetilde{E}_{j l}}-1, \quad \varphi_{j}=e^{-\beta \widetilde{E}_{j}^{0}} Z_{0}^{-1}, \quad Z_{0}=\int d \gamma e^{-\beta \widetilde{E}^{0}}
$$


Таким образом, формула (9) действительно имеет структуру классической статистической суммы, что позволяет при расчетах $Z_{N}$ применять те же методы, которые используются в классическом случае, модифицировав их должным образом. В частности, для систем с короткодействующим взаимодействием конфигурационный интеграл $Q_{N}$ из (11) можно представить в форме группового разложения. При этом для определения явного вида групповых интегралов можно воспользоваться обычной майеровской диаграммной техникой [19]. Следует только сопоставлять линиям диаграмм “функции Майера" $F_{j l}$, а вершинам - операцию усреднения с одночастичной функцией распределения $\varphi_{j}$. Тогда, например, первые два групповых интеграла представятся формулами

$$
b_{1}=\int d \gamma(1) f_{1}=1, \quad b_{2}=\frac{1}{2} \int d \gamma(1) d \gamma(2) \varphi_{1} \varphi_{2} F_{12} \equiv \frac{1}{2}\left\langle F_{12}\right\rangle_{12} .
$$

Аналогично можно обобшить соответствуюшие выражения для систем с дальнодействуюшим взаимодействием. Так, если ограничиться приближением хаотических фаз, то $Q_{N}$ можно представить в виде (см., например, [20])

$$
Q_{N}=\exp \left(\frac{1}{2} \sum_{s \geqslant 2} \frac{1}{s}(-\beta N)^{s} W_{s}\right)
$$

где величина $W_{s}$ определяет вклад кольцевой диаграммы $s$-го порядка. Подобно (13) в этом случае вкладу $W_{s}$ следует сопоставить выражение

$$
\int d \gamma(1) \ldots d \gamma(s) \varphi_{1} \ldots \varphi_{s}\left(\widetilde{E}_{12} \widetilde{E}_{23} \ldots \widetilde{E}_{s 1}\right) \equiv\left\langle\widetilde{E}_{12} \widetilde{E}_{23} \ldots \widetilde{E}_{s 1}\right\rangle_{1 \ldots s} .
$$

Проиллюстрируем предложенный метод расчета статистической суммы на примере модельной системы с лагранжианом взаимодействия Дарвина

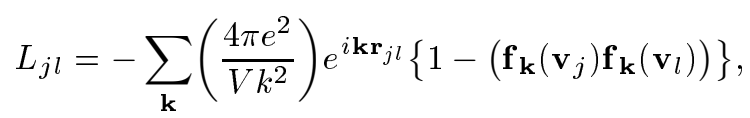

где $\mathbf{f}_{\mathbf{k}}\left(\mathbf{v}_{j}\right)=(1 / c k)\left[\mathbf{k v}_{j}\right], \mathbf{r}_{j l}=\mathbf{r}_{j}-\mathbf{r}_{l}$. В этом случае согласно (10) энергия $\widetilde{E}_{j l}$ представится формулой

$$
\begin{aligned}
\widetilde{E}_{j l} & =\sum_{\mathbf{k}}\left(\frac{4 \pi e^{2}}{V k^{2}}\right) e^{i \mathbf{k r}_{j l}}\left\{1+\left(\mathbf{f}_{\mathbf{k}}\left(\mathbf{v}_{j}\right) \mathbf{f}_{\mathbf{k}}\left(\mathbf{v}_{l}\right)\right)+\left(\mathbf{f}_{\mathbf{k}}\left(\overline{\mathbf{u}}_{j}\right) \mathbf{f}_{\mathbf{k}}\left(\mathbf{u}_{l}\right)\right)+\left(\mathbf{f}_{\mathbf{k}}\left(\overline{\mathbf{u}}_{l}\right) \mathbf{f}_{\mathbf{k}}\left(\mathbf{u}_{j}\right)\right)\right\} \equiv \\
& \equiv E_{j l}^{(1)}+E_{j l}^{(2)}+E_{j l}^{(3)}+E_{l j}^{(3)} .
\end{aligned}
$$

Здесь $E_{j l}^{(1)}$ - энергия кулоновского взаимодействия, $E_{j l}^{(2)}$ - обычная релятивистская поправка на взаимодействие, $E_{j l}^{(3)}+E_{l j}^{(3)}-$ дополнительная энергия, обусловленная введением "грассмановых скоростей". Отметим, что $E_{j l}^{(3)} \neq E_{l j}^{(3)}$.

5 Теоретическая и математическая физика, т. 126, № 2, 2001 г. 
Для свободного лагранжиана $L_{j}^{0}$ сохраним точное релятивистское выражение. Тогда

$$
\begin{gathered}
L_{j}^{0}=-m c^{2}\left(1-\frac{v_{j}^{2}}{c^{2}}\right)^{\frac{1}{2}}, \\
\widetilde{E}_{j}^{0}=\frac{m c^{2}}{\sqrt{1-\frac{v_{j}^{2}}{c^{2}}}}+\frac{m}{\sqrt{1-\frac{v_{j}^{2}}{c^{2}}}}\left[\delta_{\mu \nu}+\frac{\frac{v_{j}^{\mu} v_{j}^{\nu}}{c^{2}}}{1-\frac{v_{j}^{2}}{c^{2}}}\right] \bar{u}_{j}^{\mu} u_{j}^{\nu} .
\end{gathered}
$$

Рассмотрим кольцевую диаграмму $W_{s}$. Подставив $(17)$ в $(15)$, нетрудно увидеть, что

$$
W_{s}=\sum_{n=1}^{4} W_{s}^{(n)}
$$

где

$$
W_{s}^{(n)}=\left\langle\widetilde{E}_{12}^{(n)} \widetilde{E}_{23}^{(n)} \ldots \widetilde{E}_{s 1}^{(n)}\right\rangle_{1 \ldots s} \text { при } n=1,2,3
$$

a

$$
W_{s}^{(4)}=\left\langle\widetilde{E}_{21}^{(3)} \widetilde{E}_{32}^{(3)} \ldots \widetilde{E}_{1 s}^{(3)}\right\rangle_{1 \ldots s} .
$$

Остальные слагаемые, получающиеся после раскрытия произведения $\left(\widetilde{E}_{12} \ldots \widetilde{E}_{s 1}\right)$, содержат либо множители, линейные по скоростям $v_{j}^{\mu}$ (или по "грассмановым скоростям" $\bar{u}_{j}^{\mu}, u_{l}^{\mu}$ одной из частищ), либо квадратичные множители $u_{j}^{\mu} u_{j}^{\nu}, \bar{u}_{l}^{\mu} \bar{u}_{l}^{\nu}$ (последние содержатся в произведениях $\left.E_{j l}^{(3)} E_{i l}^{(3)}, E_{l j}^{(3)} E_{l i}^{(3)}\right)$. При усреднении такие слагаемые исчезают вследствие нечетности подынтегральных выражений по скоростям $v_{j}$ или соотношений (6). Кроме того, поскольку энергии $E_{j l}^{(3)}$ коммутируют между собой, то, изменив в члене $W_{s}^{(4)}$ нумерацию переменных интегрирования, найдем, что $W_{s}^{(4)}=W_{s}^{(3)}$. Учитывая все это, а также равенство

$$
\frac{1}{(2 \pi)^{3}} \int e^{i\left(\mathbf{k r}-\mathbf{k}^{\prime} \mathbf{r}\right)} d \mathbf{r}=V \delta_{\mathbf{k}, \mathbf{k}^{\prime}}
$$

после несложных преобразований получим

$$
\begin{gathered}
(-\beta N)^{s} W_{s}^{(1)}=\sum_{k}\left(-\frac{\kappa^{2}}{k^{2}}\right)^{s}, \\
(-\beta N)^{s} W_{s}^{(2)}=\sum_{k}\left(-\frac{\kappa^{2}}{k^{2}}\right)^{s}\left\langle\left(\mathbf{f}_{\mathbf{k}}\left(\mathbf{v}_{1}\right) \mathbf{f}_{\mathbf{k}}\left(\mathbf{v}_{2}\right)\right)\left(\mathbf{f}_{\mathbf{k}}\left(\mathbf{v}_{2}\right) \mathbf{f}_{\mathbf{k}}\left(\mathbf{v}_{3}\right)\right) \ldots\left(\mathbf{f}_{\mathbf{k}}\left(\mathbf{v}_{s}\right) \mathbf{f}_{\mathbf{k}}\left(\mathbf{v}_{1}\right)\right)\right\rangle_{1 \ldots s}= \\
=\sum_{k}\left(-\frac{\kappa^{2}}{k^{2}}\right)^{s} \Pi_{k}^{\mu_{1} \mu_{2}} \Pi_{k}^{\mu_{2} \mu_{3}} \ldots \Pi_{k}^{\mu_{s} \mu_{1}}, \\
(-\beta N)^{s} W_{s}^{(3)}=\sum_{k}\left(-\frac{\kappa^{2}}{k^{2}}\right)^{s}\left\langle\left(\mathbf{f}_{\mathbf{k}}\left(\overline{\mathbf{u}}_{1}\right) \mathbf{f}_{\mathbf{k}}\left(\mathbf{u}_{2}\right)\right)\left(\mathbf{f}_{\mathbf{k}}\left(\overline{\mathbf{u}}_{2}\right) \mathbf{f}_{\mathbf{k}}\left(\mathbf{u}_{3}\right)\right) \ldots\left(\mathbf{f}_{\mathbf{k}}\left(\overline{\mathbf{u}}_{s}\right) \mathbf{f}_{\mathbf{k}}\left(\mathbf{u}_{1}\right)\right)\right\rangle_{1 \ldots s}= \\
=-\sum_{k}\left(-\frac{\kappa^{2}}{k^{2}}\right)^{s} \widetilde{\Pi}_{k}^{\mu_{1} \mu_{2}} \widetilde{\Pi}_{k}^{\mu_{2} \mu_{3}} \ldots \widetilde{\Pi}_{k}^{\mu_{s} \mu_{1}},
\end{gathered}
$$


где

$$
\Pi_{k}^{\mu \nu}=\left\langle f_{\mathbf{k}}^{\mu}(\mathbf{v}) f_{\mathbf{k}}^{\nu}(\mathbf{v})\right\rangle_{1}, \quad \widetilde{\Pi}_{k}^{\mu \nu}=\left\langle f_{\mathbf{k}}^{\mu}(\mathbf{u}) f_{\mathbf{k}}^{\nu}(\overline{\mathbf{u}})\right\rangle_{1}, \quad \kappa^{2}=\frac{4 \pi e^{2} \beta N}{V} .
$$

Тензоры $\Pi_{k}^{\mu \nu}, \widetilde{\Pi}_{k}^{\mu \nu}$ являются функциями вектора $\mathbf{k}$. Поэтому они выражаются только через единичный тензор $\delta_{\mu \nu}$ и тензор $k_{\mu} k_{\nu}$. Учитьвая явный вид векторов $\mathbf{f}_{\mathbf{k}}$, легко убедиться, что

$$
\Pi_{k}^{\mu \nu}=e_{\mu \nu} \Pi_{k}, \quad \widetilde{\Pi}_{k}^{\mu \nu}=e_{\mu \nu} \widetilde{\Pi}_{k},
$$

где $e_{\mu \nu}=\delta_{\mu \nu}-k_{\mu} k_{\nu} / k^{2}$,

$$
\begin{aligned}
& \Pi_{k}=\frac{1}{2 c^{2}}\left\langle(\mathbf{v v})-\frac{(\mathbf{k v})^{2}}{k^{2}}\right\rangle_{1}=e_{\sigma \rho} \frac{1}{c^{2}}\left\langle v^{\sigma} v^{\rho}\right\rangle_{1}, \\
& \widetilde{\Pi}_{k}=\frac{1}{2 c^{2}}\left\langle(\mathbf{u} \overline{\mathbf{u}})-\frac{(\mathbf{k u})(\mathbf{k} \overline{\mathbf{u}})}{k^{2}}\right\rangle_{1}=e_{\sigma \rho} \frac{1}{c^{2}}\left\langle u^{\sigma} \bar{u}^{\rho}\right\rangle_{1} .
\end{aligned}
$$

Покажем, что $\Pi_{k}^{\mu \nu}=\widetilde{\Pi}_{k}^{\mu \nu}$. Для этого достаточно сравнить средние $\left\langle v^{\mu} v^{\nu}\right\rangle_{1}$ и $\left\langle u^{\mu} \bar{u}^{\nu}\right\rangle_{1}$. Они определяются выражениями

$$
\begin{aligned}
& \left\langle u^{\sigma} \bar{u}^{\rho}\right\rangle_{1}=\int d \gamma \frac{1}{Z^{0}} e^{-\beta \widetilde{E}^{0}} u^{\sigma} \bar{u}^{\rho}, \\
& \left\langle v^{\sigma} v^{\rho}\right\rangle_{1}=\int d \gamma \frac{1}{Z^{0}} e^{-\beta \widetilde{E}^{0}} v^{\sigma} v^{\rho} .
\end{aligned}
$$

Интегрирование по грассмановым переменным осушествляется на основе формул (5)$(7)$, интеграл по координате х равен объему системы $V$. Получим

$$
\begin{aligned}
& \left\langle u^{\sigma} \bar{u}^{\rho}\right\rangle_{1}=\frac{V}{(2 \pi \hbar)^{3} Z^{0} \beta} \int d \mathbf{v} e^{-\beta E^{0}(\mathbf{v})} \operatorname{det}\left|\frac{\partial^{2} L^{0}}{\partial v^{\mu} \partial v^{\nu}}\right|\left(\frac{\partial^{2} L^{0}}{\partial v^{\sigma} \partial v^{\rho}}\right)^{-1}, \\
& \left\langle v^{\sigma} v^{\rho}\right\rangle_{1}=\frac{V}{(2 \pi \hbar)^{3} Z^{0}} \int d \mathbf{v} e^{-\beta E^{0}(\mathbf{v})} \operatorname{det}\left|\frac{\partial^{2} L^{0}}{\partial v^{\mu} \partial v^{\nu}}\right| v^{\sigma} v^{\rho}, \quad E^{0}(\mathbf{v})=\mathbf{v} \frac{\partial L^{0}}{\partial \mathbf{v}}-L^{0} .
\end{aligned}
$$

Поскольку, как это следует из преобразований Лежандра, вторые производные от гамильтоновой и лагранжевой функций связаны соотношением

$$
\frac{\partial^{2} L}{\partial v^{a} \partial v^{b}} \frac{\partial^{2} H}{\partial p^{b} \partial p^{c}}=\delta_{a c}
$$

при $v^{a}=\partial H / \partial p^{a}$ или $p^{a}=\partial L / \partial v^{a}$, то, выполняя в (21) замену переменных $v^{\mu}=$ $\partial H^{0} / \partial p^{\mu}$ и интегрируя по частям, будем иметь

$$
\begin{aligned}
\left\langle u^{\sigma} \bar{u}^{\rho}\right\rangle_{1} & =\frac{V}{(2 \pi \hbar)^{3} Z^{0}} \frac{1}{\beta} \int d \mathbf{p} e^{-\beta H^{0}(p)} \frac{\partial^{2} H^{0}}{\partial p^{\sigma} \partial p^{\rho}}= \\
& =\frac{V}{(2 \pi \hbar)^{3} Z^{0}} \int d \mathbf{p} e^{-\beta H^{0}(p)} \frac{\partial H_{0}}{\partial p^{\sigma}} \frac{\partial H_{0}}{\partial p^{\rho}} .
\end{aligned}
$$


Нетрудно убедиться, что такой же результат получается и для среднего $\left\langle v^{\mu} v^{\nu}\right\rangle_{1}$. Использовав это в формулах (20), видим, что $\Pi_{k}=\widetilde{\Pi}_{k}$, т.е. $\Pi_{k}^{\mu \nu}=\widetilde{\Pi}_{k}^{\mu \nu}$. Последнее равенство не зависит от конкретного вида величин $\widetilde{E}^{0}, L^{0}, H^{0}$. В нашем случае $L_{0}, \widetilde{E}^{0}$ oпределяются формулами (18), а свободный гамильтониан $H^{0}(p)=\left(m^{2} c^{4}+c^{2} p^{2}\right)^{1 / 2} \equiv \varepsilon(p)$. Тогда из соотношений (20)-(22) следует, что

$$
\Pi_{k}=\left\langle\frac{c^{2} p^{2}}{3 \varepsilon^{2}(p)}\right\rangle^{0} \equiv \delta, \quad \Pi_{k}^{\mu \nu}=e_{\mu \nu} \delta
$$

где $\langle\ldots\rangle^{0}$ обозначает усреднение с релятивистским распределением Максвелла.

Учитывая приведенные выше результаты и то, что $e_{\mu_{1} \mu_{2}} e_{\mu_{2} \mu_{3}}=e_{\mu_{1} \mu_{3}}, e_{\mu \mu}=2$, получим

$$
(-\beta N)^{s} W_{s}=\sum_{k}\left(-\frac{\kappa^{2}}{k^{2}}\right)^{s}-2 \sum_{k}\left(-\frac{\kappa^{2} \delta}{k^{2}}\right)^{s} .
$$

Подставив это соотношение в формулу (14), найдем, что поправка на взаимодействие к свободной энергии $\Delta F=-\beta^{-1} \ln Q_{N}$ рассматриваемой здесь модельной системы определяется выражением

$$
\Delta F=-\frac{1}{2 \beta} \sum_{k}\left[\frac{\kappa^{2}}{k^{2}}-\ln \left(1+\frac{\kappa^{2}}{k^{2}}\right)\right]+\frac{1}{\beta} \sum_{k}\left[\frac{\kappa^{2} \delta}{k^{2}}-\ln \left(1+\frac{\kappa^{2} \delta}{k^{2}}\right)\right] .
$$

Выполнив суммирование, получим

$$
\Delta F=-\frac{V \kappa^{3}}{12 \pi \beta}\left(1-2 \delta^{\frac{3}{2}}\right) \equiv \Delta F^{\mathrm{c}}+\Delta F^{\mathrm{r}} .
$$

Здесь $\Delta F^{\mathrm{c}}$ определяет вклад кулоновского взаимодействия, а $\Delta F^{\mathrm{r}}$ - релятивистскую поправку, обусловленную магнитным взаимодействием при точном учете в нем релятивистских кинематических эффектов. В слаборелятивистском приближении последние не учитьваются. Поэтому, как видно из $(23), \delta \simeq\left\langle p^{2} / 3 m^{2} c^{2}\right\rangle^{0}=1 / \beta m c^{2}=\delta^{\prime}$. Тогда

$$
\Delta F^{\mathrm{r}}=\frac{V}{6 \pi \beta}\left(\frac{4 \pi N e^{2}}{V m c^{2}}\right)^{\frac{3}{2}}
$$

что совпадает с известным результатом работы [7]. Таким образом, релятивистская поправка к свободной энергии пропорциональна $(e / c)^{3}$. Этот результат соответствует общей специфике статистических систем с дальнодействующим взаимодействием, термодинамические функции которых неаналитичны по константе взаимодействия. В нашем случае в релятивистской части лагранжиана взаимодействия (16) скорость света фигурирует в сочетании типа $e^{2} / c^{2}$ с константой взаимодействия $e^{2}$. Поэтому естественно, что свободная энергия оказывается неаналитичной по параметру $e^{2} / c^{2}$.

В заключение рассмотрим результаты, которые получаются при вычислении статистической суммы (2) в канонических переменных. 
1. Если для гамильтоновой функции воспользоваться приближением (1), то, приняв во внимание (16), найдем, что релятивистская часть взаимодействия представится функцией

$$
H_{j l}^{\mathrm{r}}=-\sum_{k} \frac{4 \pi e^{2}}{V k^{2}} e^{i \mathbf{k r}_{j l}} c^{2}\left(\mathbf{f}_{\mathbf{k}}\left(\mathbf{p}_{j}\right) \mathbf{f}_{\mathbf{k}}\left(\mathbf{p}_{l}\right)\right) \varepsilon^{-1}\left(p_{j}\right) \varepsilon^{-1}\left(p_{l}\right)
$$

Она отличается знаком от выражения для $E_{j l}^{(2)}$ формулы (17), которое описывает релятивистское взаимодействие в лагранжевых переменных. Поэтому среднее $\left\langle H_{12}^{\mathrm{r}} \ldots H_{s 1}^{\mathrm{r}}\right\rangle$, определяюшее вклад кольцевой диаграммы, будет содержать дополнительный множитель $(-1)^{s}$. Тогда, выполнив надлежашие расчеты, можно показать, что

$$
(-\beta N)^{s} W_{s}^{\mathrm{r}}=2 \sum_{k} \frac{1}{s}\left(\frac{\kappa^{2}}{k^{2}} \delta\right)^{s}
$$

где число $\delta$ то же, что и в (24). Таким образом, релятивистская часть вклада кольцевых диаграмм представится расходяшимся рядом

$$
\sum_{k} \sum_{s \geqslant 2} \frac{1}{s}\left(\frac{\kappa^{2}}{k^{2}} \delta\right)^{s}
$$

2. Если же при определении гамильтониана $H$ в (1) учесть поправки высшего порядка, то вместо (25) получим гамильтониан, содержащий многочастичные взаимодействия. В приближении хаотических фаз их можно аппроксимировать эффективным двухчастичным взаимодействием, имеюшим экранированный характер [9]. Кроме того, при такой аппроксимации перенормируется также масса частицы:

$$
m \rightarrow m^{*}=m\left(1-\frac{V \kappa^{2}\left(\delta^{\prime}\right)^{\frac{3}{2}}}{6 \pi N}\right)
$$

Вследствие экранирования вместо упомянутого выше ряда получим сходяшееся выражение

$$
\sum_{k} \sum_{s \geqslant 2} \frac{1}{s}\left(\frac{\kappa^{2} \delta^{\prime}}{k^{2}+\kappa^{2} \delta^{\prime}}\right)^{s}=\frac{1}{12 \pi} V \kappa^{3}\left(\delta^{\prime}\right)^{\frac{3}{2}}
$$

Учитывая, что параметр $\kappa^{2} \delta^{\prime}$ содержится также в члене $Z^{0}\left(m^{*}\right)$, получим для релятивистской поправки выражение, совпадаюшее с результатом работ [7], [9]. Естественно, что применять эффективный гамильтониан можно только в приближении хаотических фаз. Его нельзя использовать, например, для вычисления вклада вириальных коэффициентов в статистическую сумму слаборелятивистской плазмы. В этом отношении преимушество введения в теорию грассмановых переменных очевидно. 


\section{Список литературы}

[1] Л. Д. Ландау, Е. М. Лифииц. Теория поля. М.: Наука, 1973.

[2] F. D. Kennedy. Amer. J. Phys. 1972. V. 40. № 1. P. 63.

[3] Р. П. Гайда. ЭЧАЯ. 1982. Т. 13. № 2. С. 427.

[4] Л. Ф. Блажиевський, Г. Б. Гіль, С. С. Семак. Журн. фіз. досл. 1996. Т. 1. № 1. С. 1.

[5] J.E. Krizan, P. Havas. Phys. Rev. 1962. V. 128. № 12. P. 2916.

[6] J. E. Krizan. Phys. Rev. A. 1965. V. 140. № 5. P. 1155.

[7] Б. А. Трубников, В. П. Косачев. ЖЭТФ. 1968. Т. 54. № 3. С. 939.

[8] J. E. Krizan. Phys. Rev. A. 1974. V. 10. № 1. P. 298.

[9] Л. Ф. Блажиевский. Укр. физ. журн. 1975. Т. 20. № 8. С. 1273.

[10] R. D. Jones, A. Pytte. Phys. Fluids. 1980. V. 23. № 3. P. 269.

[11] R. Lapiedra, E. Santos. Phys. Rev. D. 1981. V. 23. № 10. P. 269.

[12] J. E. Krizan. Phys. Rev. D. 1982. V. 25. № 2. P. 593.

[13] X. Barcons, R. Lapiedra. Phis. Rev. A. 1983. V. 28. № 3. P. 3030.

[14] И. М. Павлоцкий. Введение в слаборелятивистскую статистическую механику. М.: Ин-т прикладной математики, 1987.

[15] H. Essen. Phys. Rev. E. 1997. V. 56. № 5. P. 5858.

[16] H. Essen. J. Phys. A. 1999. V. 32. P. 2297.

[17] Л. Ф. Блажиевский. ТМФ. 1986. Т. 66. № 3. С. 409.

[18] Ф. А. Березин. Метод вторичного квантования. М.: Наука, 1965.

[19] Джс. Майер, М. Гепперт-Майер. Статистическая механика. М.: Мир, 1980.

[20] А. Исихара. Статистическая физика. М.: Мир, 1973.

Поступила в редакцию $18 . \mathrm{IV} .2000$ г. после доработки 10.VIII.2000 г. 